\title{
The Usefulness and Limitations of Impedance Cardiography for Cardiac Resynchronization Therapy Device Optimization
}

\author{
Kojiro Ogawa, ${ }^{1,2}$ MD, Miyako Igarashi, ${ }^{1} \mathrm{MD}$, Akihiko Nogami, ${ }^{1} \mathrm{MD}$, Masayoshi Yamamoto, ${ }^{1} \mathrm{MD}$, \\ Akinori Sugano, ${ }^{1,3}$ MD, Yukio Sekiguchi ${ }^{1,4}$ MD, Kazutaka Aonuma, ${ }^{1}$ MD and Masaki Ieda, ${ }^{1}$ MD
}

\begin{abstract}
Summary
Identifying the optimal atrioventricular (AV) or interventricular (VV) delay is beneficial for patients using cardiac resynchronization therapy (CRT) devices. Ultrasonic echocardiography (UCG) has been the most commonly used method; however, it requires high technical knowledge. Impedance cardiography (ICG) can calculate stroke volume by measuring changes in transthoracic electric impedance. This study sought to assess the clinical utility of ICG in comparison with that of UCG for the optimization of CRT devices.

Patients who underwent CRT device implantation were retrospectively analyzed. One week after implantation, optimization of AV delay (AVD) was performed in every patient with ICG (AVD-ICG) and UCG (AVDUCG). VV delay (VVD) was then determined according to the optimal AVD using these two methods.

Forty-two patients were enrolled. Average AVD-ICG was significantly shorter than AVD-UCG $(128 \pm 49$ versus $146 \pm 41$ milliseconds, $P=0.018)$. Five patients $(12 \%)$ had the same optimized AVD with two methods, and the difference between AVD-ICG and AVD-UCG was $\leq 20$ milliseconds in 19 patients (45\%). In the multivariate analysis, the presence of postoperative mitral regurgitation (MR) was an independent predictor of AVDICG/AVD-UCG mismatch, defined as a difference over 20 milliseconds (odds ratio $=10.71 ; 95 \%$ confidence interval $=1.72$ to $66.72 ; P=0.018$ ). The results of optimized VVD were similar using both methods.

ICG might be a promising tool for the rapid optimization of CRT devices. However, in patients with moderate-to-severe MR, ICG may not be able to optimize AVD.
\end{abstract}

(Int Heart J 2020; 61: 896-904)

Key words: Atrioventricular delay, Interventricular delay, Noninvasive hemodynamic assessment, Cardiac pacing, Mitral regurgitation

$\mathrm{P}$ atients with heart failure frequently exhibit symptoms and signs of the condition even after optimal medical treatment. ${ }^{1}$ Cardiac resynchronization therapy (CRT) has been used in such patients with dyssynchrony. The first CRT devices were indicated for patients with New York Heart Association (NYHA) functional class III or IV heart failure. ${ }^{2-4)}$ Currently, CRT devices are widely indicated even in patients with milder symptoms and sufficient intraventricular or interventricular (VV) conduction delay. ${ }^{5-8)}$ To identify the optimal atrioventricular (AV) or VV intervals may result in an improvement in clinical outcomes. ${ }^{910)}$ However, optimization of CRT devices is not adequately performed in many cases. ${ }^{11)}$

Automated periodical optimization algorithms of AV delay (AVD) and/or VV delay (VVD) have been developed and are now available in various CRT devices. These new algorithms were reported to be safe and effective in improving cardiac performance. ${ }^{12)}$ Because of these automated processes, the transition of AVDs/VVDs could be overlooked by doctors and other healthcare professionals.
Therefore, it would be important to validate the settings manually, especially in cases of poor hemodynamic conditions. Although invasive measurement remains the gold standard to optimize these intervals, noninvasive methods would be preferable on an outpatient basis. Ultrasonic echocardiography (UCG) has been reported as a reliable tool to optimize AVD in patients with CRT devices. ${ }^{13)}$ However, it was reported to have low validity in the clinical setting, ${ }^{14)}$ where there is currently no alternative to UCG.

Impedance cardiography (ICG) can calculate stroke volume (SV) and cardiac output (CO) by measuring changes in the transthoracic electric impedance of a lowamplitude, high-frequency current constantly applied via body surface electrodes. The SV and $\mathrm{CO}$ estimated by ICG were reported to be reliable when compared with those measured with invasive methods. ${ }^{15,16)}$ ICG might constitute an easy and useful noninvasive technique to optimize the AVD in patients with pacemakers ${ }^{17)}$ and those with CRT devices. ${ }^{18,19)}$ However, the usefulness of ICG for

From the ${ }^{1}$ Department of Cardiology, Faculty of Medicine, University of Tsukuba, Tsukuba, Japan, ${ }^{2}$ Department of Cardiology, Hitachi General Hospital, Hitachi, Japan, ${ }^{3}$ Department of Cardiology, Tsukuba Medical Center Hospital, Tsukuba, Japan and ${ }^{4}$ Department of Internal Medicine (Cardiology), Kasumigaura Medical Center, Tsuchiura, Japan.

Address for correspondence: Miyako Igarashi, MD, Department of Cardiology, Faculty of Medicine, University of Tsukuba, 1-1-1 Tennodai, Tsukuba, Ibaraki 305-8575, Japan. E-mail: migarashi@md.tsukuba.ac.jp

Received for publication November 11, 2019. Revised and accepted April 30, 2020.

doi: 10.1536/ihj.19-620

All rights reserved by the International Heart Journal Association. 
Table I. How to Optimize Cardiac Resynchronization Therapy Devices with Impedance Cardiography (ICG)

\begin{tabular}{|c|c|c|c|c|c|c|c|c|c|c|c|c|}
\hline \multirow{3}{*}{$\begin{array}{l}\text { AVD (milliseconds) } \\
\text { VVD (milliseconds) }\end{array}$} & \multicolumn{8}{|c|}{ AVD optimization } & \multicolumn{4}{|c|}{ VVD optimization } \\
\hline & 60 & 80 & 100 & 120 & 140 & 160 & 180 & 200 & \multicolumn{4}{|c|}{100 (fixed) } \\
\hline & \multicolumn{8}{|c|}{0 (fixed) } & 0 & 20 & 40 & 60 \\
\hline HR (bpm) & 64 & 64 & 64 & 64 & 66 & 69 & 68 & 68 & 64 & 64 & 66 & 69 \\
\hline $\mathrm{SV}(\mathrm{mL})$ & 59 & 61 & 61 & 59 & 57 & 54 & 54 & 52 & 61 & 58 & 54 & 54 \\
\hline $\mathrm{CO}(\mathrm{L} /$ minute $)$ & 3.8 & 3.9 & 3.9 & 3.8 & 3.8 & 3.7 & 3.7 & 3.5 & 3.9 & 3.7 & 3.6 & 3.7 \\
\hline
\end{tabular}

The AVD was adjusted starting from 100 milliseconds and shortened or prolonged stepwise by 20 milliseconds during simultaneous biventricular pacing (VVD $=0$ milliseconds). In this representative case, the maximum stroke volume was $61 \mathrm{~mL}$, which was attained during pacing with AVDs of 80 and 100 milliseconds. In such cases, the longer or the longest AVD was used as the optimized interval. Therefore, 100 milliseconds was chosen as the optimal AVD (AVD-ICG). Next, the VVD was also adjusted from RV-LV simultaneous pacing to LV pre-excitation in incremental steps of 20 milliseconds under the fixed optimal AVD until the estimated SV decreased repeatedly. In this case, the maximum SV was recorded under the VVD of 0 milliseconds. Therefore, the optimal VVD (VVD-ICG) was 0 milliseconds. AVD indicates atrioventricular delay; CO, cardiac output; CRT, cardiac resynchronization therapy; HR, heart rate; SV, stroke volume; and VVD, interventricular delay.

CRT optimization has only been shown in limited settings, and direct comparisons of ICG with invasive measurements have only been performed under certain conditions. A comparison between ICG and UCG in various device modes or settings is helpful to assess the usefulness of ICG for the optimization of CRT devices.

This study aimed to evaluate the use of ICG as an optimizing tool for CRT devices in comparison with echoDoppler optimization of AVDs/VVDs.

\section{Methods}

Study subjects: We examined consecutive patients with heart failure who underwent successful implantation of a CRT device at the University of Tsukuba and Tsukuba Medical Center Hospital between January 2014 and the end of 2016. Every patient provided written informed consent for device implantation at each hospital. All patients were in NYHA class II, III, or IV after receiving guideline-directed medical therapy for at least 3 months. Patients received CRT devices according to a standard class I indication for heart failure with a reduced left ventricular (LV) systolic function with a LV ejection fraction $<35 \%$ and QRS duration $\geq 120$ milliseconds. ${ }^{6}$ Cases of upgrading pacemakers to CRT devices or implantable cardioverter defibrillators (ICD) to CRT defibrillator (CRT-D) were included. Patients with persistent atrial fibrillation ( $n$ $=5$ ), high burden of ventricular premature beats up to $\geq$ $10 \%(n=3)$, hemodialysis $(n=1)$, and failing to carry out optimization with ICG $(n=4)$ were excluded. The CRT pacemakers (CRT-Ps) and CRT-Ds used in this study were Viva $^{\mathrm{TM}}$ CRT-P (Medtronic Inc., Minneapolis, MN, USA), Viva ${ }^{\mathrm{TM}}$ XT CRT-D (Medtronic Inc., Minneapolis, MN, USA), or Quadra Assura ${ }^{\text {TM }}$ CRT-D (St. Jude Medical, St. Paul, MN, USA).

One week after implantation, all patients underwent optimization of their CRT device with both ICG and UCG. We compared the optimized AVDs using ICG (AVD-ICG) and UCG (AVD-UCG), as well as the tailored VVDs using ICG (VVD-ICG) and UCG (VVD-UCG). We defined the difference in optimized AVDs ( $\triangle \mathrm{AVD})$ or VVDs $(\triangle \mathrm{VVD})$ in these two methods as $(\triangle \mathrm{AVD}=[\mathrm{AVD}-$ ICG] - [AVD-UCG], $\Delta$ VVD = [VVD-ICG] - [VVDUCG]). Negative $\triangle$ AVD values implied AVD-ICG was shorter than AVD-UCG. Negative $\triangle$ VVD indicated that
LV was paced earlier during pacing using UCG than when using ICG. Patients were divided into two groups: the matched group and the mismatched group. If $\triangle \mathrm{AVD}$ was $\leq 20$ milliseconds, patients were classified into the AVD-matched group. If $\triangle \mathrm{AVD}$ was $>20$ milliseconds, they were classified into the AVD-mismatched group. Grouping into the VVD-matched and VVD-mismatched groups was performed according to $\triangle \mathrm{VVD}$ in the same way.

During the follow-up period, the automatic and periodical optimization of the AV or VV interval was applied if the device allowed it. If not, primary physicians chose the $\mathrm{AV}$ and $\mathrm{VV}$ intervals optimized in one of these two methods at the time of discharge.

The study was approved by the Institutional Review Board at each hospital.

Optimization with ICG: The ICG system used in this study was a commercially available device (Task Force Monitor Systems, CN Systems. Austria), ${ }^{20)}$ which calculates $\mathrm{SV}$ beat by beat and $\mathrm{CO}$ by measuring changes in the transthoracic electric impedance of a low-amplitude, high-frequency current constantly applied via surface electrodes placed on the upper left and right anterolateral chest wall near the diaphragm in combination with one electrode at the posterior cervical region. The result shown in Table I is a representative case we have done. First, we optimized AVD under a VVD of 0 milliseconds. VVD optimization was then employed under the fixed AVD initially determined. The measurement time was 90 seconds at each setting, which is longer than that of previous studies $^{19)}$ to identify consistent values for SV. AV interval was adjusted in steps of 20 milliseconds. Second, VVD was adjusted from zero (right ventricle-LV simultaneous pacing) to LV pre-excitation using 20 milliseconds steps unless estimated SV showed decreased reproducibly under the fixed AVD at which highest SV was first measured.

If the same result of SV was obtained in different AVDs, we decided the longer or the longest AVD as the best in order to maximize the atrial kick effect. If the same SV result was obtained with a different VVD, we repeated the test until the differences were observed in a reproducible fashion.

Optimization with UCG: Optimization with UCG was performed within 7 days after implantation and 24 hours before or after optimization with ICG. All echocardiograms were performed by well-trained doctors who were 
not informed of the results of optimization by ICG. Commercially available ultrasound machines (EPIQ 7C, Philips Medical Systems, Best, The Netherlands) or Vivid 7 (General Electric, Milwaukee, WI, USA) were used. Doppler transmitral and LV outflow tract evaluation was performed. The transmitral flow was obtained in the fourchamber view with the sample positioned at the tip of the mitral leaflets. First, the AVD was optimized using either Ritter's method or the iterative method. ${ }^{21,22)}$ We programmed AVD as long as possible until neither diastolic mitral regurgitation (MR) nor manifest fusion of $\mathrm{E}$ and $\mathrm{A}$ waves was observed. AVDs were then shortened by 20milliseconds increments under monitoring pulsed-wave Doppler transmitral inflow until truncation of the A wave was noted. The AVD-UCG was identified by progressively lengthening the AVD until A wave truncation was no longer present, and the $\mathrm{E}$ and $\mathrm{A}$ waves were not superimposed. Second, the VV interval was adjusted with visual evaluation and time-velocity integral (TVI) at the LV outflow tract. The VV timing with the highest TVI was recorded and defined as VVD-UCG. ${ }^{23)}$

Statistical analysis: Continuous variables are expressed as mean \pm standard deviation or median [interquartile range (IQR)]. Differences between the two optimization methods in optimized AVD and electrocardiographic parameters, including QRS width, were evaluated by paired t-tests. Categorical variables were compared using chi-square or Fisher's exact tests.

\section{Results}

Patient characteristics: The baseline characteristics of 42 patients (age, $68 \pm 12$ years), including 10 cases $(23 \%)$ of the upgrade from an ICD to a CRT-D $(n=4)$ or a pacemaker to a CRT-D $(n=6)$ are summarized in Table II. All patients had a QRS duration > 130 milliseconds in the baseline ECG. Optimization with ICG and UCG was performed on a median of 6.9 days (range 5-9 days) and 6.2 days (range 4-10 days) after implantation, respectively. Among the 27 patients who were implanted with a device capable of periodical optimization of AVD and VVD, automated adjustment was performed in all patients at first but discontinued in two patients during follow-up due to significant discrepancies between automatically optimized AVD or VVD with a device and those manually optimized with ICG or UCG. In these two patients, AVD-UCG and VVD-UCG were ultimately decided according to the physician's judgment. In the remaining 15 patients whose device was not capable of periodic optimization automatically, AV or VVD was fixed to the interval optimized by either ICG or UCG. Two patients had the same optimization results in two methods in both AV and VVD, and the AVD-ICG and VVD-ICG or AVD-UCG and VVD-UCG intervals were set according to the physician's preference in seven and eight patients, respectively.

Comparison of optimized AVDs: AVD-ICG and AVDUCG were the same in only 5 patients $(12 \%)$; 19 patients $(45 \%)$ had optimized AVDs with a difference of $\leq 20$ milliseconds between methods (Figure 1A). Another 16 patients $(38 \%)$ had a shorter AVD-ICG than AVD-UCG, and 7 patients $(17 \%)$ had longer AVD-ICG than AVD-UCG.
Mean AVD-ICG was significantly shorter than mean AVD-UCG $(128 \pm 49$ versus $146 \pm 41$ milliseconds, $P=$ $0.018)$. A simple regression analysis revealed a mild positive correlation between AVD-ICG and AVD-UCG $(r=$ $0.48, P=0.001$, Figure 1B).

A significant correlation was observed between AVDICG and AVD-UCG $(r=0.52, P=0.003)$ when focusing on only the patients with no or mild MR after CRT $(n=$ 30, Figure 2A). However, there was no significant correlation between AVD-ICG and AVD-UCG in patients with moderate-to-severe MR (ms-MR) $(n=12)(r=0.39, P=$ 0.21 , Figure 2B). On the other hand, averaged AVD-ICG was still shorter than AVD-UCG, even in patients without ms-MR after CRT $(131 \pm 46$ versus $148 \pm 41$ milliseconds, $P<0.001)$.

Comparison of optimized VVDs: The results of optimization of VVD were similar using both ICG and UCG (0 [0 to 20] versus 20 [0 to 20] milliseconds, $P=0.15$, Figure $3 \mathrm{~A})$. Thirty-seven patients $(88 \%)$ had similar optimized VVDs with both ICG and UCG. Furthermore, VVD-ICG and VVD-UCG were the same in 19 (45\%) patients (Figure 3A). Simple regression analysis revealed a mild positive correlation between VVD-ICG and VVDUCG ( $r=0.51, P=0.001$, Figure 3B).

Factors related to the mismatch between ICG and UCG optimization: As for the VVD, 37 patients (88\%) were classified into the VVD-matched group. We compared clinical characteristics between the VVD-matched and VVD-mismatched groups. However, no statistical difference was observed between groups.

On the other hand, 19 patients $(45 \%)$ who had similar AVD-UCG and AVD-ICG results were classified into the AVD-matched group. The remaining 23 patients $(55 \%)$ were classified into the AVD-mismatched group (Table II). There was no difference in baseline characteristics between groups. However, the prevalence of postoperative ms-MR was significantly higher in the AVD-mismatched group ( $11 \%$ versus $43 \%, P=0.037)$.

In addition, we divided the patients in the AVDmismatched group into two groups: the patients who had shorter AVD-ICG than AVD-UCG $(n=16)$ and the patients with longer AVD-ICG than AVD-UCG $(n=7)$. The comparison between the two groups demonstrated that patients with shorter AVD-ICG were significantly younger than those with longer AVD-ICG $(62 \pm 12$ versus $75 \pm 9$, $P=0.018)$. However, there was no significant difference in other factors between groups (Table III).

MR and a mismatch of optimized AVDs: As indicated by red-colored bars or markers in Figure 1A, B and 2B, postoperative ms-MR was significantly associated with a mismatch between AVD-ICG and AVD-UCG with good specificity (sensitivity $43 \%$, specificity $90 \%$, positive predictive value $83 \%$, and negative predictive value $57 \%$ ).

In univariate analysis, details described in Table IV, postoperative ms-MR was found to be a significant predictor of AVD-ICG/AVD-UCG mismatch and ms-TR was a possible predictor of this mismatch although statistically insignificant (ms-MR, odds ratio (OR) 6.54; 95\% confidence interval (CI) 1.22 to $35.1 ; P=0.029$, ms-TR, OR 3.72; 95\% CI 0.670-20.6, $P=0.13$ ). 
Table II. Baseline Characteristics and Differences between AVD-Matched and Mismatched Groups

\begin{tabular}{|c|c|c|c|c|}
\hline & $\begin{array}{c}\text { All } \\
n=42\end{array}$ & $\begin{array}{c}\text { AVD matched } \\
n=19\end{array}$ & $\begin{array}{c}\text { AVD mismatched } \\
n=23\end{array}$ & $P$ value \\
\hline Age (years) & $68 \pm 12$ & $71 \pm 8$ & $66 \pm 12$ & 0.13 \\
\hline Male gender, $n(\%)$ & $28(67 \%)$ & $12(63 \%)$ & $16(70 \%)$ & 0.75 \\
\hline $\operatorname{BSA}\left(\mathrm{cm}^{2}\right)$ & $1.61 \pm 0.19$ & $1.58 \pm 0.21$ & $1.63 \pm 0.17$ & 0.35 \\
\hline \multicolumn{5}{|l|}{ NYHA, $n(\%)$} \\
\hline II & $14(33 \%)$ & $6(32 \%)$ & $8(35 \%)$ & 1.00 \\
\hline III & $26(62 \%)$ & $12(63 \%)$ & $14(61 \%)$ & 1.00 \\
\hline IV & $2(5 \%)$ & $1(5 \%)$ & $1(4 \%)$ & 1.00 \\
\hline \multicolumn{5}{|l|}{ Etiology, $n(\%)$} \\
\hline ICM & $12(29 \%)$ & $6(32 \%)$ & $6(26 \%)$ & 0.74 \\
\hline DCM & $18(43 \%)$ & $8(42 \%)$ & $10(43 \%)$ & 1.00 \\
\hline $\mathrm{HCM}$ & $2(5 \%)$ & $1(5 \%)$ & $1(4 \%)$ & 1.00 \\
\hline Sarcoidosis & $8(19 \%)$ & $4(21 \%)$ & $4(17 \%)$ & 1.00 \\
\hline Others & $2(5 \%)$ & $0(0 \%)$ & $2(9 \%)$ & 0.49 \\
\hline \multicolumn{5}{|l|}{ Comorbidities, $n(\%)$} \\
\hline HT & $19(45 \%)$ & $10(53 \%)$ & $10(43 \%)$ & 0.76 \\
\hline $\mathrm{DM}$ & $19(45 \%)$ & $8(42 \%)$ & $12(52 \%)$ & 0.55 \\
\hline COPD & $8(19 \%)$ & $4(21 \%)$ & $4(17 \%)$ & 1.00 \\
\hline CKD & $26(62 \%)$ & $12(63 \%)$ & $14(61 \%)$ & 1.00 \\
\hline \multicolumn{5}{|c|}{ ECG findings } \\
\hline \multicolumn{5}{|c|}{ QRSd (milliseconds) } \\
\hline Pre & $165 \pm 31$ & $163 \pm 29$ & $169 \pm 34$ & 0.54 \\
\hline Post & $150 \pm 25$ & $145 \pm 19$ & $155 \pm 29$ & 0.16 \\
\hline RA paced, $n(\%)$ & $24(57 \%)$ & $10(53 \%)$ & $14(61 \%)$ & 0.54 \\
\hline \multicolumn{5}{|l|}{ Echocardiography } \\
\hline $\operatorname{LVEF}(\%)$ & $31 \pm 9(12-44)$ & $31 \pm 8$ & $30 \pm 9$ & 0.62 \\
\hline LVEDV (mm) & $63 \pm 8(49-90)$ & $163 \pm 60$ & $186 \pm 67$ & 0.26 \\
\hline $\mathrm{LAD}(\mathrm{mm})$ & $42 \pm 7(18-53)$ & $42 \pm 6$ & $42 \pm 8$ & 0.93 \\
\hline \multicolumn{5}{|l|}{ ms-MR, $n(\%)$} \\
\hline Pre & $14(33 \%)$ & $4(21 \%)$ & $10(43 \%)$ & 0.19 \\
\hline Post & $12(29 \%)$ & $2(11 \%)$ & $10(43 \%)$ & 0.037 \\
\hline \multicolumn{5}{|l|}{ ms-TR, $n(\%)$} \\
\hline Pre & $13(31 \%)$ & $5(26 \%)$ & $8(35 \%)$ & 0.74 \\
\hline Post & $9(21 \%)$ & $2(11 \%)$ & $7(30 \%)$ & 0.15 \\
\hline \multicolumn{5}{|l|}{ ms-AR, $n(\%)$} \\
\hline Pre & $2(5 \%)$ & $1(5 \%)$ & $1(4 \%)$ & 1.00 \\
\hline Post & $2(5 \%)$ & $1(5 \%)$ & $1(4 \%)$ & 1.00 \\
\hline \multicolumn{5}{|l|}{ Medication, $n(\%)$} \\
\hline ACE-I/ARB & $34(81 \%)$ & $17(89 \%)$ & $17(74 \%)$ & 0.26 \\
\hline $\mathrm{BB}$ & $36(86 \%)$ & $17(89 \%)$ & $19(83 \%)$ & 0.67 \\
\hline MRA & $30(71 \%)$ & $15(80 \%)$ & $15(79 \%)$ & 0.50 \\
\hline Amiodarone & $25(60 \%)$ & $9(47 \%)$ & $16(70 \%)$ & 0.21 \\
\hline \multicolumn{5}{|l|}{ Blood examination } \\
\hline Cre $(\mathrm{mg} / \mathrm{dL})$ & $1.3 \pm 0.8(0.5-5.1)$ & $1.3 \pm 0.8$ & $1.3 \pm 0.9$ & 0.93 \\
\hline T-bil (mg/dL) & $1.0 \pm 0.7(0.3-3.8)$ & $0.8 \pm 0.5$ & $1.2 \pm 0.9$ & 0.12 \\
\hline $\mathrm{BNP}(\mathrm{pg} / \mathrm{mL})$ & $390[197-980]$ & 252 [203-818] & $460[190-1021]$ & 0.86 \\
\hline $\mathrm{Hb}(\mathrm{g} / \mathrm{dL})$ & $12.7 \pm 2.3$ & $12.7 \pm 2.6$ & $12.7 \pm 2.1$ & 0.99 \\
\hline
\end{tabular}

ACE-I indicates angiotensin-converting enzyme inhibitor; (ms-) AR, (moderate-to-severe) aortic valve regurgitation; ARB, angiotensin-II receptor blocker; BB, beta blocker; BNP, B-type (brain) natriuretic peptide; BSA, body surface area; CKD, chronic kidney disease; COPD, chronic obstructive pulmonary disease; Cre, creatinine; DCM, dilated cardiomyopathy; DM, diabetes mellitus; Hb, hemoglobin; HCM, hypertrophic cardiomyopathy; HT, hypertension; ICM, ischemic cardiomyopathy; LAD, left atrial diameter; LBBB, left bundle branch block; LVEDV, left ventricular end-diastolic volume; LVEF, left ventricular ejection fraction; (ms-) MR, (moderate-to-severe) mitral valve regurgitation; MRA, mineralocorticoid-receptor antagonist; NYHA, New York Heart Association functional class; QRSd, QRS duration; RA, right atrium; T-bil, total bilirubin; and (ms-) TR, (moderate-to-severe) tricuspid valve regurgitation.

\section{Discussion}

Major findings: This study investigated the reliability of ICG as a noninvasive method of CRT optimization in comparison with UCG. The main findings are that (1) the optimized AVD-ICG was significantly shorter than AVDUCG, (2) no significant difference was observed between VVD-ICG and VVD-UCG, and (3) patients with ms-MR after CRT device implantation tend to have a difference in AVD optimization results using the two methods (OR 

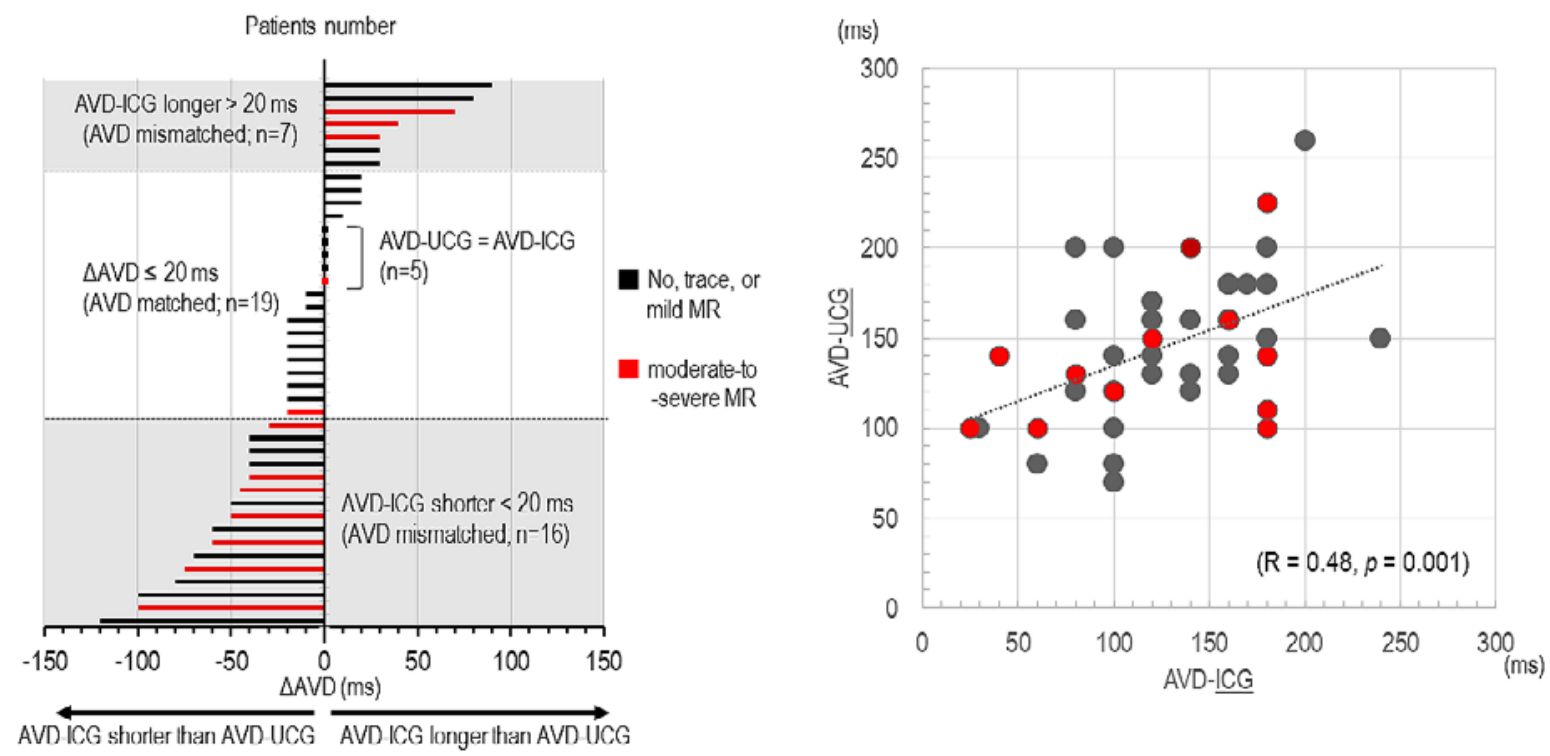

Figure 1. A: The difference of the AV delays optimized with ICG (AVD-ICG) and those with UCG (AVD-UCG). $\triangle A V D$ with negative figures implied AVD-ICG were shorter than AVD-UCG. Nineteen (45\%) patients had similar ( $<20$ milliseconds) optimized AV delays. More than half of the patients had longer AVD-UCG than AVD-ICG. The patients with postoperative moderate-to-severe MR (red bar) were related to mismatch between AVD-ICG and AVD-UCG. B: The mild correlation between AVD-UCG and AVD-ICG was observed $(r=0.48, P=0.001)$.

A

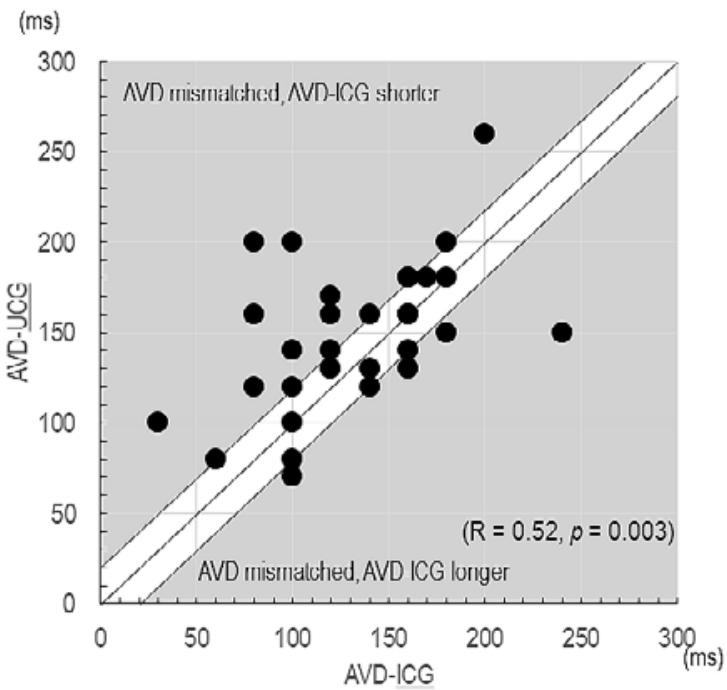

B

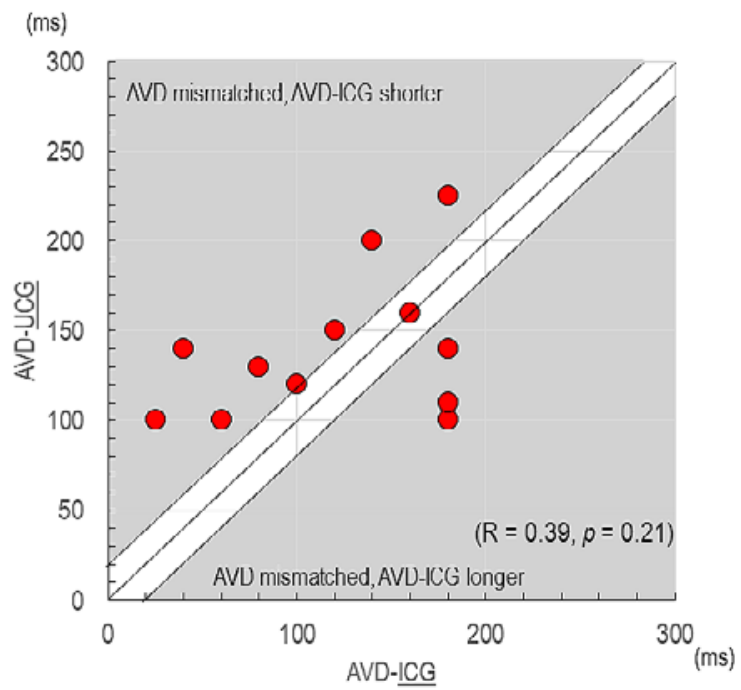

Figure 2. A: Better correlation was observed between AVD-ICG and AVD-UCG when focusing on only the patients with no or mild MR after CRT $(n=30)(r=0.52, P=0.003)$. B: No significant correlation in patients with moderate-to-severe MR $(n=12)(r=0.39, P=0.21)$.

6.54).

Usefulness of AVD/VVD optimization: Achieving optimal outcomes from CRT is thought to be dependent on AVD optimization. ${ }^{24)}$ VVD optimization would also be important for hemodynamic improvement in patients with CRT devices according to the results of the InSync III study, which found an increase in SV by individualizing the optimal VV interval from simultaneous biventricular pacing to sequential CRT. ${ }^{25}$ Combining optimized AVDs/ VVDs provided the best hemodynamic response. ${ }^{26}$

Problems of AVD/VVD optimization with UCG: Choosing a method for AVD/VVD optimization is a crucial matter. UCG is the most widely used method; however, one study demonstrated that the optimization of AVD and 
A

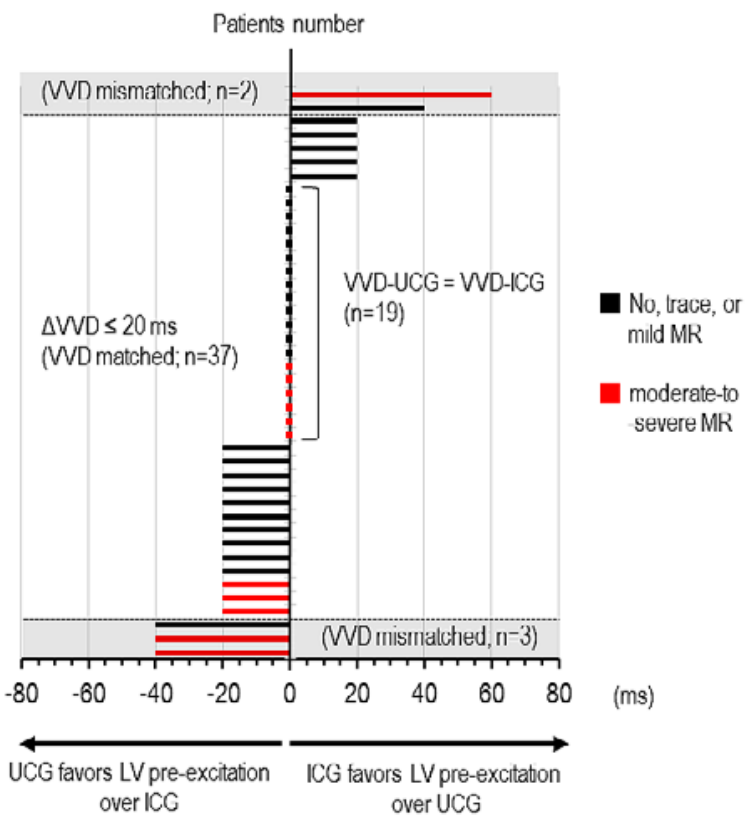

B

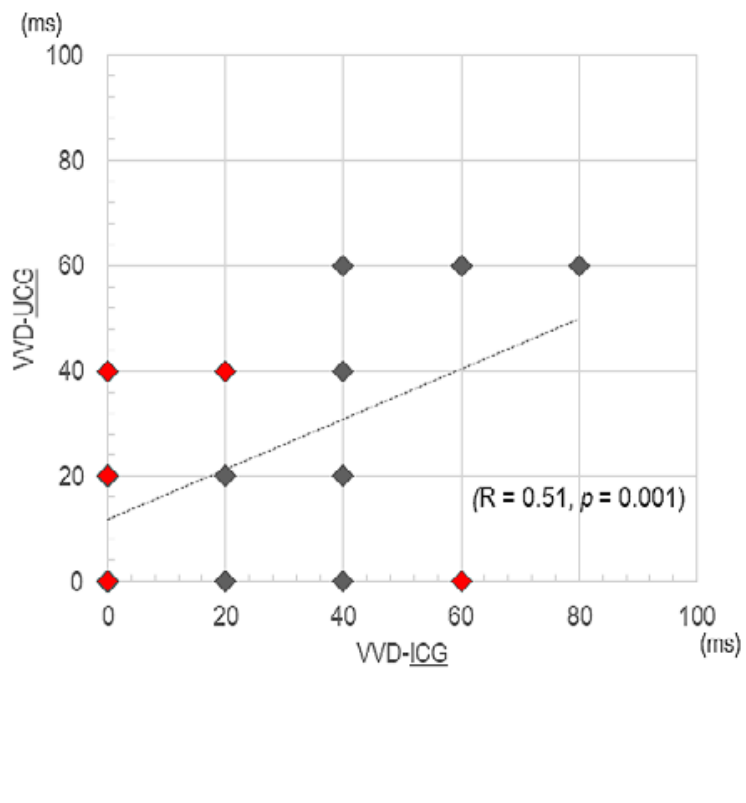

Figure 3. A: ICG-optimized VV delays and those with UCG were completely matched in 19 patients (45\%), and nearly matched (the difference $<20$ milliseconds) in 37 patients $(88 \%)$. B: The mild correlation was observed between VVD-UCG and VVD-ICG $(r=0.51, P=0.001)$.

VVD with UCG failed to significantly improve SV. ${ }^{27}$

Technical problems when using UCG are, to a certain degree, inevitable. For instance, a quantitative assessment of VV dyssynchrony is sometimes challenging. Methodological problems can also be a concern. For optimization of AVD using UCG, we used the inflow-based Doppler echocardiography techniques, which have been reported previously ${ }^{2,13)}$ and are predominantly used in the clinical setting. However, the problem with this method is that only preload optimization is achieved, and the apparent non-preload determinants of aortic pulse pressure are not accounted for. A previous study pointed out that the optimum AVD was sometimes shorter than the AVD obtained using the inflow Doppler technique because the minimum AV mechanical latency would correspond to the time of peak velocity in the A wave and not to the end of the A wave. ${ }^{24)}$

Advantages of ICG-guided optimization: ICG is a noninvasive and simple method that only requires the placement of planar electrodes on the trunk and posterior neck. As previously reported, there were no significant differences in SV and CO when using ICG, thermodilution, and direct Fick. ${ }^{15,16)}$ Most previous studies comparing the determination of CO using ICG and invasive methods have shown a correlation of 0.7 to 0.9 , and the accuracy of determining $\mathrm{CO}$ using ICG could be affected by its inherent limitations as well as by pathological states, including too low or high $\mathrm{CO}$, intracardiac shunts, valvular regurgitation, and arrhythmias. ${ }^{28)}$

Factors influencing AVD optimization differences between ICG and UCG: To identify the factors influencing the mismatch between AVD-ICG and AVD-UCG is important for clinical practice. Regarding the AVD, previous studies showed that optimized AVDs determined by ICG were comparable with those measured by UCG, with a very strong correlation between the two. ${ }^{18,27)}$ However, only a mild correlation between AVD-ICG and AVD-UCG was observed in the present study, and the average AVDICG was shorter than the AVD-UCG.

There are several possibilities that could explain this difference. Notably, we included both patients with RA pacing $(n=19)$ and RA sensing $(n=23)$ in the present study. On the other hand, all patients were examined either in sinus rhythm ${ }^{18)}$ or at a fixed atrio-biventricular stimulation $^{27)}$ in previous studies. Patients with RA sensing would have increased heart rate variability, which might lead to a larger beat-by-beat change in thoracic impedance and instability of SV estimation with ICG.

More importantly, ms-MR after CRT device implantation was the most significant contributing factor to the difference between AVD-ICG and AVD-UCG in our study.

The shorter ICG-optimized AVD compared with that using UCG is a matter of concern; a possible mechanism is the increase in forward output with the decrease of regurgitant volume; that is, the decrease of the mitral regurgitant fraction (RF) could be achieved using a shorter $\mathrm{AVD}$, as reported before. ${ }^{29)}$

What is considered the best is also an important problem.

Concerning UCG-guided AVD optimization, Ritter's method and the iterative method we used in this study are not the ways to calculate forward output directly. Total $\mathrm{SV}$, that is, the summation of the forward and the regurgitant SVs, could be maximized with these methods. The forward output might be increased simultaneously in many cases; however, it could be decreased if mitral RF 
Table III. Comparison between Shorter Optimized AVD with ICG (AVD-ICG) and Longer AVD-ICG Groups in Patients with Mismatched AVD

\begin{tabular}{|c|c|c|c|}
\hline & $\begin{array}{c}\text { AVD-ICG shorter } \\
n=16\end{array}$ & $\begin{array}{c}\text { AVD-ICG longer } \\
n=7\end{array}$ & $P$ value \\
\hline Age (years) & $62 \pm 12$ & $75 \pm 9$ & 0.018 \\
\hline Male gender, $n(\%)$ & $11(69 \%)$ & $5(71 \%)$ & 1.00 \\
\hline BMI $\left(\mathrm{kg} / \mathrm{m}^{2}\right)$ & $23.3 \pm 3.2$ & $21.1 \pm 1.8$ & 0.12 \\
\hline Ischemic cardiomyopathy & $3(19 \%)$ & $3(43 \%)$ & 0.32 \\
\hline \multicolumn{4}{|l|}{ Comorbidities, $n(\%)$} \\
\hline Hypertension & $6(38 \%)$ & $4(57 \%)$ & 0.65 \\
\hline Diabetes & $7(58 \%)$ & $5(71 \%)$ & 0.37 \\
\hline COPD & $2(13 \%)$ & $2(29 \%)$ & 0.56 \\
\hline CKD & $9(56 \%)$ & $5(71 \%)$ & 0.66 \\
\hline \multicolumn{4}{|l|}{ ECG findings } \\
\hline QRS duration, pre CRT (milliseconds) & $174 \pm 32$ & $159 \pm 38$ & 0.33 \\
\hline QRS duration, post CRT (milliseconds) & $163 \pm 29$ & $139 \pm 23$ & 0.068 \\
\hline Right atrium paced, $n(\%)$ & $6(38 \%)$ & $3(43 \%)$ & 1.00 \\
\hline \multicolumn{4}{|l|}{ Echocardiography (pre) } \\
\hline $\operatorname{LVEF}(\%)$ & $28 \pm 9$ & $35 \pm 7$ & 0.10 \\
\hline LVEDV (mm) & $149 \pm 28$ & $203 \pm 74$ & 0.078 \\
\hline $\begin{array}{l}\mathrm{LAD}(\mathrm{mm}) \\
\mathrm{ms}-\mathrm{MR}, n(\%)\end{array}$ & $42 \pm 6$ & $39 \pm 10$ & 0.32 \\
\hline Pre & $7(44 \%)$ & $3(43 \%)$ & 1.00 \\
\hline Post & $7(44 \%)$ & $3(43 \%)$ & 1.00 \\
\hline \multicolumn{4}{|l|}{ ms-TR, $n(\%)$} \\
\hline Pre & $6(38 \%)$ & $2(29 \%)$ & 1.00 \\
\hline Post & $5(31 \%)$ & $2(29 \%)$ & 1.00 \\
\hline \multicolumn{4}{|l|}{ Medication, $n(\%)$} \\
\hline ACE-I/ARB & $10(63 \%)$ & $7(100 \%)$ & 0.12 \\
\hline Beta-blocker & $14(88 \%)$ & $5(71 \%)$ & 0.35 \\
\hline MRA & $11(69 \%)$ & $4(57 \%)$ & 0.47 \\
\hline amiodarone & $12(75 \%)$ & $4(57 \%)$ & 0.63 \\
\hline \multicolumn{4}{|l|}{ Blood examination } \\
\hline Cre $(\mathrm{mg} / \mathrm{dL})$ & $1.3 \pm 1.1$ & $1.3 \pm 0.6$ & 1.00 \\
\hline T-bil (mg/dL) & $1.2 \pm 1.0$ & $1.1 \pm 0.8$ & 0.81 \\
\hline $\mathrm{BNP}(\mathrm{pg} / \mathrm{mL})$ & 415 [202-989] & $573[169-1021]$ & 0.86 \\
\hline $\mathrm{Hb}(\mathrm{g} / \mathrm{dL})$ & $13.0 \pm 1.2$ & $12.0 \pm 1.8$ & 0.30 \\
\hline
\end{tabular}

ACE-I indicates angiotensin-converting enzyme inhibitor; (ms-) AR, (moderate-to-severe) aortic valve regurgitation; ARB, angiotensin-II receptor blocker; $\mathrm{BB}$, beta blocker; BNP, B-type (brain) natriuretic peptide; BSA, body surface area; CKD, chronic kidney disease; COPD, chronic obstructive pulmonary disease; Cre, creatinine; DCM, dilated cardiomyopathy; DM, diabetes mellitus; $\mathrm{Hb}$, hemoglobin; HCM, hypertrophic cardiomyopathy; HT, hypertension; ICM, ischemic cardiomyopathy; ICG, impedance cardiography; LAD, left atrial diameter; LBBB, left bundle branch block; LVEDV, left ventricular end-diastolic volume; LVEF, left ventricular ejection fraction; (ms-) MR, (moderate-to-severe) mitral valve regurgitation; MRA, mineralocorticoid-receptor antagonist; NYHA, New York Heart Association functional class; QRSd, QRS duration; RA, right atrium; T-bil, total bilirubin; and (ms-) TR, (moderate-to-severe) tricuspid valve regurgitation.

was increased. In this regard, direct calculation of the forward SV would be ideal to optimize the AVD, especially in cases with ms-MR.

On the other hand, ICG optimization would have some methodological problems.

We should be aware that it is not possible to speculate either the forward or the regurgitant SV with ICG selectively. Theoretically, the regurgitant flow does not contribute changes in the volume and velocity of the blood flow in the thoracic aorta, which means that the presence of MR has no or little effect on ICG. However, it was previously reported that the waveform of impedance cardiogram could be changed depending on the presence of regurgitant flow. ${ }^{30)}$ In this report, correlation between the severity of MR and the waveform changes of ICG was also pointed out. A better correlation between $\mathrm{CO}$ values estimated using ICG and UCG was observed in patients without MR compared with those with MR in another study. ${ }^{31)}$

If MR had some effects on ICG, MR in diastole could not be negligible; hence, we cannot detect diastolic MR with ICG. As a result, diastolic MR could be present under AVD-ICG. It is uncertain whether the effect of diastolic regurgitant flow to ICG is subtle enough to ignore in clinical settings. It would be possible that some AVDICGs might be misguided because of diastolic MR, especially those in patients who had much longer AVD-ICG than AVD-UCG.

Moreover, several approximations and assumptions upon which ICG-guided estimation of SV stands on could 
Table IV. Factors Related to the Mismatch between ICG and Echocardiography Optimization by Univariate Analysis

\begin{tabular}{lccc}
\hline & Odds ratio & $95 \%$ CI & $P$ value \\
\hline Age, per 10-year increase & 0.697 & $0.386-1.26$ & 0.23 \\
Sex, male category & 1.33 & $0.368-4.83$ & 0.75 \\
BSA, per 0.1 cm ${ }^{2}$ increase & 5.01 & $0.180-14.0$ & 0.34 \\
NYHA functional class IV & 0.818 & $0.048-14.0$ & 0.89 \\
Etiology, $n$ (\%) & & & \\
ICM & 0.765 & $0.200-2.93$ & 0.70 \\
DCM & 1.06 & $0.310-3.61$ & 0.93 \\
HCM & 0.818 & $0.048-14.0$ & 0.89 \\
Comorbidities, $n$ (\%) & & & \\
HT & 0.692 & $0.204-2.35$ & 0.56 \\
DM & 1.50 & $0.441-5.10$ & 0.52 \\
COPD & 0.789 & $0.169-3.69$ & 0.76 \\
CKD & 0.907 & $0.259-3.18$ & 0.88 \\
ECG & & & \\
QRSd, per 10 milliseconds increase & 1.02 & $0.992-1.05$ & 0.15 \\
RA paced & 0.579 & $0.169-1.98$ & 0.38 \\
Echocardiography & & & \\
LVEF, per 10\% increase & 0.979 & $0.506-1.89$ & 0.95 \\
LVEDV, per 10 mL increase & 1.01 & $0.995-1.02$ & 0.25 \\
LAD, per 10 mm increase & 0.908 & $0.806-1.02$ & 0.12 \\
Postoperative msMR & 6.54 & $1.22-35.1$ & 0.029 \\
Postoperative msTR & 3.72 & $0.670-20.6$ & 0.13 \\
Postoperative msAR & 0.818 & $0.048-14.0$ & 0.89 \\
Blood examination & & & \\
Log BNP, per 1 increase & 0.988 & $0.375-2.61$ & 0.98 \\
Hb, per 1 g/dL increase & 1.00 & $0.770-1.30$ & 1.00 \\
\hline
\end{tabular}

Abbreviations are listed in Table II.

be inadequate. One of them is that the adults' thorax acts as a cylinder or a truncated cone and it is homogeneously perfused with blood. We believe hemodynamic homogeneity would not always be acceptable in certain cases, particularly in cases with AV valve regurgitation. Indeed, no good correlation was observed between AVD-ICG and AVD-UCG in patients with ms-MR after CRT in our study (Figure 2B). Turbulence blood flow in atria by regurgitant flow and two blood volumes moving in the two different directions, that is, regurgitant blood volume and antegrade blood volume in the descending aorta, would cause local inhomogeneity and lead to a mismatch between true SV and estimated SV. According to the results of the univariate analysis, both MR and TR are possible contributing factors, although statistically insignificant. The number of patients with postoperative ms-TR was smaller than that of patients with ms-MR, and the turbulence effects generated by TR would be weaker than those generated by MR as the trans-tricuspid pressure gradient during systole is generally smaller than the trans-mitral pressure gradient. These two would make ms-TR statistically and hemodynamically underpowered.

To the best of our knowledge, no report was available concerning the clinical feasibility of ICG for CRT optimization with sufficient information about preoperative and/or postoperative valvular heart disease and their severity.

Of note, there were seven patients with ms-MR who had longer AVD-ICG than AVD-UCG values. As shown in Table III, younger age may be related to a shorter AVD-
ICG. Therefore, unidentified factors would also increase the difference between AVD-ICG and AVD-UCG.

Limitations: This study has several limitations. First, the sample size in this study was relatively small. Second, no direct comparison was made between ICG and invasive SV estimation. However, SV calculated with ICG has been shown to be similar to SV evaluated using invasive methods. ${ }^{15,16)}$ Finally, clinical outcomes were not evaluated in this study. Therefore, we cannot make any inferences regarding prognosis. Further research, such as casecrossover studies or randomized control studies, would be necessary to decide which optimized AVDs/VVDs delivers a better prognosis.

\section{Conclusions}

ICG is a noninvasive monitoring tool to optimize the settings of CRT devices that could substitute UCG. In VVD optimization, ICG could substitute UCG as the results were similar between the two methods. However, there was a significant difference in AVD optimization results between ICG and UCG, especially in patients with ms-MR after CRT.

\section{Disclosure}

Conflicts of interest: Dr. Aonuma belongs to the endowed department of Boston Scientific, Japan, and Biotronik Japan. Dr. Nogami belongs to the endowed de- 
partment of Medtronic. Dr. Sekiguchi belonged to the endowed department of Abbott Japan.

\section{References}

1. Cleland JG, Clark AL. Delivering the cumulative benefits of triple therapy to improve outcomes in heart failure: too many cooks will spoil the broth. J Am Coll Cardiol 2003; 42: 1234-7.

2. Cazeau S, Leclercq C, Lavergne T, et al. Effects of multisite biventricular pacing in patients with heart failure and intraventricular conduction delay. N Engl J Med 2001; 344: 873-80.

3. Abraham WT, Fisher WG, Smith AL, et al. Cardiac resynchronization in chronic heart failure. N Engl J Med 2002; 346: 184553.

4. Young JB, Abraham WT, Smith AL, et al. Combined cardiac resynchronization and implantable cardioversion defibrillation in advanced chronic heart failure: the MIRACLE ICD Trial. JAMA 2003; 289: 2685-94

5. Moss AJ, Hall WJ, Cannom DS, et al. Cardiacresynchronization therapy for the prevention of heart-failure events. N Engl J Med 2009; 361: 1329-38.

6. Tang AS, Wells GA, Talajic M, et al. Cardiac-resynchronization therapy for mild-to-moderate heart failure. N Engl Med 2010; 363: 2385-95.

7. Goldenberg I, Kutyifa V, Klein HU, et al. Survival with cardiacresynchronization therapy in mild heart failure. N Engl J Med 2014; 370: 1694-701.

8. Chen K, Su H, Xie C, et al. Prognostic implications of QRS duration in third-degree atrioventricular block patients with heart failure treated with cardiac resynchronization therapy. Int Heart J 2018; 59: 1320-6.

9. Auricchio A, Stellbrink C, Block M, et al. Effect of pacing chamber and atrioventricular delay on acute systolic function of paced patients with congestive heart failure. Circulation 1999; 99: 2993-3001

10. Saxon LA, Ellenbogen KA. Resynchronization therapy for the treatment of heart failure. Circulation 2003; 108: 1044-8.

11. Gras D, Gupta MS, Boulogne E, Guzzo L, Abraham WT. Optimization of $\mathrm{AV}$ and $\mathrm{VV}$ delays in the real-world CRT patient population: an international survey on current clinical practice. Pacing Clin Electrophysiol 2009; 32: S236-9.

12. Martin DO, Lemke B, Birnie D, et al. Investigation of a nove algorithm for synchronized left-ventricular pacing and ambulatory optimization of cardiac resynchronization therapy: results of the adaptive CRT trial. Heart Rhythm 2012; 9: 1807-14.

13. Kerlan JE, Sawhney NS, Waggoner AD, et al. Prospective comparison of echocardiographic atrioventricular delay optimization methods for cardiac resynchronization therapy. Heart Rhythm 2006; 3: 148-54

14. Raphael CE, Kyriacou A, Jones S, et al. Multinational evaluation of the interpretability of the iterative method of optimisation of AV delay for CRT. Int J Cardiol 2013; 168: 407-13.

15. Belardinelli R, Ciampani N, Costantini C, Blandini A, Purcaro A. Comparison of impedance cardiography with thermodilution and direct Fick methods for noninvasive measurement of stroke volume and cardiac output during incremental exercise in patients with ischemic cardiomyopathy. Am J Cardiol 1996; 77 1293-301.

16. Drazner $\mathrm{MH}$, Thompson B, Rosenberg PB, et al. Comparison of impedance cardiography with invasive hemodynamic measurements in patients with heart failure secondary to ischemic or nonischemic cardiomyopathy. Am J Cardiol 2002; 89: 993-5.

17. Ovsyshcher I, Zimlichman R, Katz A, Bondy C, Furman S. Measurements of cardiac output by impedance cardiography in pacemaker patients at rest: effects of various atrioventricular delays. J Am Coll Cardiol 1993; 21: 761-7.

18. Braun MU, Schnabel A, Rauwolf T, Schulze M, Strasser RH. Impedance cardiography as a noninvasive technique for atrioventricular interval optimization in cardiac resynchronization therapy. J Interv Card Electrophysiol 2005; 13: 223-9.

19. Heinroth KM, Elster M, Nuding S, et al. Impedance cardiography: A useful and reliable tool in optimization of cardiac resynchronization devices. Europace 2007; 9: 744-50.

20. Fortin J, Haitchi G, Bojic A. Validation and verification of the Task Force ${ }^{\circledR}$ Monitor. Results of clinical studies for FDA 2001; 510: 014063.

21. Gorcsan J III, Abraham T, Agler DA, et al. Echocardiography for cardiac resynchronization therapy: recommendations for performance and reporting-a report from the American Society of Echocardiography Dyssynchrony Writing Group endorsed by the Heart Rhythm Society. J Am Soc Echocardiogr 2008; 21: 191-213.

22. Kedia N, Ng K, Apperson-Hansen C, et al. Usefulness of atrioventricular delay optimization using Doppler assessment of mitral inflow in patients undergoing cardiac resynchronization therapy. Am J Cardiol 2006; 98: 780-5.

23. Gassis S, León AR. Cardiac resynchronization therapy: strategies for Device Programming, Troubleshooting and Follow-Up. J Interv Card Electrophysiol 2005; 13: 209-22.

24. Auricchio A, Ding J, Spinelli JC, et al. Cardiac resynchronization therapy restores optimal atrioventricular mechanical timing in heart failure patients with ventricular conduction delay. J Am Coll Cardiol 2002; 39: 1163-9.

25. León AR, Abraham WT, Brozena S, et al. Cardiac resynchronization with sequential biventricular pacing for the treatment of moderate-to-severe heart failure. J Am Coll Cardiol 2005; 46: 2298-304.

26. Urbanek B, Chudzik M, Klimczak A, Rosiak M, Lewek J, Wranicz JK. Whether noninvasive optimization of AV and VV delays improves the response to cardiac resynchronization therapy. Cardiol J 2013; 20: 411-7.

27. Turcott RG, Witteles RM, Wang PJ, Vagelos RH, Fowler MB, Ashley EA. Measurement precision in the optimization of cardiac resynchronization therapy. Circ Heart Fail 2010; 3: 395404.

28. Ovsyshcher I, Furman S. Impedance cardiography for cardiac output estimation in pacemaker patients: review of the literature. Pacing Clin Electrophysiol 1993; 16: 1412-22.

29. Rossi R, Muia N, Turco V, Sgura FA, Molinari R, Modena MG. Short atrioventricular delay reduces the degree of mitral regurgitation in patients with a sequential dual-chamber pacemaker. Am J Cardiol 1997; 80: 901-5.

30. Schieken RM, Patel MR, Falsetti HL, Lauer RM. Effect of mitral valvular regurgitation on transthoracic impedance cardiogram. Br Heart J 1981; 45: 166-72.

31. van der Meer NJ, Vonk Noordegraaf A, Kamp O, de Vries PM. Noninvasive measurement of cardiac output: two methods compared in patients with mitral regurgitation. Angiology 1999; 50: 95-101. 